\title{
Escherichia coli portador de betalactamasas de espectro extendido. Resistencia
}

\author{
Miranda García $\mathrm{M}^{\mathrm{a}} \mathrm{C} .{ }^{1}$ \\ Sanid. mil. 2013; 69 (4): 244-248; ISSN: 1887-8571
}

\begin{abstract}
RESUMEN
Introducción: Escherichia coli es el microorganismo que con más frecuencia se encuentra implicado en infecciones nosocomiales y comunitarias, patógeno responsable en la etiología de infecciones de vías respiratorias altas, infecciones del tracto urinario, heridas quirúrgicas, sangre o gastroenteritis. En los últimos años ha experimentado importantes cambios encontrándose un aumento de infecciones por cepas de éstos microorganismos productores de betalactamasas de espectro extendido. Objetivos: Se decide hacer este estudio retrospectivo de las muestras procesadas en el Laboratorio de Microbiología del Hospital Básico de la Defensa San Carlos (San Fernando), para conocer la frecuencia y el patrón de sensibilidad en nuestra población por gérmenes productores de betalactamasas de espectro extendido en este caso por Escherichia coli, dada la importancia de las infecciones causadas por esta bacteria y la repercusión que tiene por todo el mundo los mecanismos de resistencia. Material y Método: Se recogieron los datos de resultados obtenidos en las muestras procesadas en el Laboratorio de Microbiología durante 36 meses (Enero 2009 a Diciembre 2011), en las que se hubieran identificado cepas de Escherichia coli y de éstas las productoras de betalactamasas de espectro extendido. Resultados: Se aislaron 34 cepas de Escherichia coli productoras de betalactamasas de espectro extendido lo que supone una tasa del 5,10\%. Se encontró una frecuencia mayor en el año 2010 (6,9\%) que en el 2009 (2,61\%), pero similar al 2011 (5,98\%). Conclusión: La frecuencia de cepas Escherichia coli con betalactamasas de espectro extendido encontrada es similar a la de otros estudios realizados en España, pero la tasa de resistencia de algunos antimicrobianos como Amoxicilina/clavulánico, Cotrimoxazol y Fluorquinolonas en nuestra población es elevada.
\end{abstract}

PALABRAS CLAVE: Escherichia coli, Betalactamasas de espectro extendido, Resistencia.

Extended spectrum beta-lactamase - positive Escherichia coli. Resistance

SUMMARY: Introduction: Escherichia coli is the microorganism most frequently found in nosocomial and community infections, responsible for upper airway infections, urinary tract infections, surgical wounds, blood infections or gastroenteritis. In the last years there have been important changes with a significant increase of infections caused by these extended spectrum beta-lactamase - positive germs. Objectives: It was decided to carry out this retrospective study of the samples processed in the microbiology laboratory of the Defense Basic Hospital «San Carlos» (San Fernando) in order to determine the frequency and sensitivity pattern of extended spectrum beta-lactamase - positive strains of E. coli, given the importance of the infections produced by this bacteria and the impact worldwide of the resistance mechanisms. Material and Method: The data obtained from the samples processed in the microbiology laboratory, and in which extended spectrum beta-lactamase - positive strains of E. coli were identified, were collected during 36 months (January 2009 to December 2011). Results: 34 extended spectrum beta-lactamase- positive strains of E. coli were isolated, that is a rate of $5.10 \%$. A higher frequency was found in the year $2010(6.9 \%)$ than in $2009(2.61 \%)$ but similar to 2011 (5.98\%). Conclusion: The frequency of extended spectrum beta-lactamase- positive strains of E. coli was similar to that found in other studies in Spain, but for some antimicrobials as amoxicillin/ clavulanic, cotrimoxazol, fluorquinolones, in our population the resistance rate is high.

KEY WORDS: Escherichia coli, Extended spectrum beta-lactamase, Resistance.

\section{INTRODUCCION}

El aumento de la resistencia bacteriana es un hecho evidente a la vez que un problema antiguo y aún cuando haya nuevos antibióticos disponibles, la presencia de microorganismos multirresistentes es cada vez más frecuente ${ }^{1}$. Escherichia coli (E. coli) es el agente etiológico más frecuente en infecciones del tracto

${ }^{1}$ Técnico Especialista de Laboratorio. Hospital General Básico de la Defensa San Carlos. Laboratorio de Microbiología. San Fernando. Cádiz. España.

Dirección para correspondencia: $M^{\mathrm{a}}$ Caridad Miranda García. Laboratorio de Microbiología. Hospital General Básico de la Defensa San Carlos. C/ Capitán Conforto s/n. San Fernando 11100 - Cádiz.

$\overline{\text { Recibido: } 26}$ de abril de 2013

Aceptado: 19 de septiembre de 2013 urinario y una de las principales causas de meningitis neonatal, además de ocasionar infecciones intestinales y otras como neumonías nosocomiales, colecistitis, peritonitis, osteomielitis, artritis infecciosa, e incluso otitis externa, siendo también una de las causas más frecuente de bacteriemia ${ }^{2}$. El aislamiento de cepas E. coli con betalactamasas de espectro extendido (BLEE) tanto en la comunidad como en el hospital se ha convertido en un problema creciente.

Las betalactamasas de espectro extendido (BLEE), también llamadas betalactamasas de espectro ampliado (BLEA), son enzimas producidas por bacilos gran negativos fundamentalmente enterobacterias, con más frecuencia por E. coli y Klebsiella pneumoniae. Son capaces de inactivar además de a las penicilinas y a las cefalosporinas de primera y segunda generación, a las oximino-cefalosporinas y al aztreonam. El primer aislamiento 


\section{Escherichia coli portador de betalactamasas de espectro extendido. Resistencia}

tuvo lugar en Alemania en 1983 a partir de una cepa de Klebsiella ozaenae recibiendo el nombre de SHV-2. En España la primera se describió en 1988. Las BLEE «clásicas» derivan de las betalactamasas de amplio espectro del grupo 2b (TEM-1, TEM2, y SHV-1) de la clasificación de Bush, Jacoby y Medeiros; poseen actividad penicilinasa y pueden ser inhibidas por el ácido clavulánico. Éstas están correlacionadas con la clasificación de Ambler $^{3}$. TEM en referencia a Temoniera nombre de la paciente en cuyo hemocultivo se aisló por primera vez una cepa $E$. coli productora de esta enzima, y SHV «sulphydril variable» que describe las propiedades bioquímicas de la enzima ${ }^{1}$. Actualmente en España está adquiriendo gran importancia un nuevo tipo de BLEE, las cefotaximasas CTX-M de naturaleza plasmídica que confieren resistencia a cefotaxima, cefuroxima y cefepime, prácticamente sin incrementar las CMI (concentración mínima inhibitoria) de la ceftazidima ${ }^{4}$.

E. coli puede producir enzimas betalactamasas cromosómicas o extracromosómicas (mediadas por plásmidos) 5 . Las cepas productoras de BLEE confieren resistencia a los betalactámicos excepto a las cefamicinas y a los carbapenémicos; pero además los plásmidos que codifican las BLEE portan genes de resistencia (transposones) a otros antimicrobianos como aminoglucósidos, tetraciclinas y cotrimoxazol, es por lo que el fenómeno de resistencia cruzada es muy frecuente y el tratamiento de las infecciones producidas por estas cepas tiene una mayor dificultad. Además, las cepas productoras de BLEE son más resistentes a fluorquinolonas que las que no lo son ${ }^{3}$.

Los betalactámicos son antibióticos de acción bactericida que actúan sobre la fase final de síntesis del peptidoglicano. Los mecanismos de resistencia a betalactámicos están mediados por:

1) Alteración de la diana (PBP) proteína fijadora de penicilina.

2) Disminución de la permeabilidad.

3) Mecanismo de eflujo o expulsión del antibiótico.

4) Inactivación enzimática por betalactamasas: cromosómicas y plasmídicas de espectro extendido ${ }^{1}$.

Actualmente padecemos la resistencia bacteriana extendida por todo el mundo.

Debido quizás al uso excesivo de antibióticos y muchas veces inadecuado, estudios realizados reconocen que han contribuido a la emergencia de la resistencia bacteriana. Las infecciones causadas por bacilos gram-negativos productores de BLEE pueden ser graves e incluso mortales. Entre los factores de riesgo en infecciones causadas por cepas productoras de BLEE se encuentran: a) la gravedad del paciente, b) duración de la hospitalización, c) intervenciones, d) catéteres-sondaje urinario, e) tratamiento antibiótico durante períodos prolongados.

Y como factores de riesgo asociados se encuentran: a) hospitalización previa, b) tratamiento antibiótico previo, c) diabetes, d) infección urinaria recurrente 5 .

El tubo digestivo es un importante reservorio de estos microorganismos, constituyendo un nicho ecológico para la transmisión de resistencia interespecie. Entre otros reservorios se encuentran las heridas colonizadas y la orofaringe. Los principales vectores de la infección son las manos entre personas de contacto estrecho, sobre todo las de los profesionales sanitarios, y como elementos implicados: termómetros, geles empleados en ecografías, sondas de oxigenoterapia o jabón líquido³ . La preva- lencia de cepas BLEE es un problema mundial. En Europa hay un importante aumento, habiendo diferencias entre distintos países, regiones e incluso centros sanitarios de una misma localidad. En España actualmente la frecuencia de cepas $E$. coli con BLEE se encuentra entre el 5-10\%, variando el porcentaje según la zona geográfica; y aproximadamente un $60 \%$ es de procedencia extrahospitalaria y de infecciones urinarias ${ }^{6}$.

El cultivo es el principal modo de demostrar le presencia de cepas $E$. coli con BLEE y poder instaurar el tratamiento correcto evitando con ello el aumento de fracasos terapéuticos.

\section{MATERIAL Y METODO}

Se recogieron los datos de resultados de las muestras procesadas en el laboratorio de Microbiología durante los años 2009, 2010 y 2011 en las que se aislaron cepas de E. coli, y de éstas las cepas productoras de BLEE. Estas muestras procedían de orinas, exudados: vaginales, óticos y faríngoamigdalares; heridas quirúrgicas, hemocultivos, catéteres, esputo y líquidos biliares.

Se sembraron según el protocolo de trabajo propio del Laboratorio. Para la identificación de las cepas y la determinación de la sensibilidad antimicrobiana se utilizó el sistema automático Microscan (Dade Behring), Panel NUC Type 52 y Neg Combo Panel Type 53; y para las cepas cuyos resultados fueron positivos a la presencia de BLEE, se siguió las normas de NCCLS ( $\mathrm{Na}$ tional Committee for Clinical Laboratory Standards) y se confirmó con el método de doble difusión disco-placa de Mueller Hinton con los antibióticos Cefotaxima y Ceftazidima con y sin Acido clavulánico, comprobándose un incremento de $5 \mathrm{~mm}$ de diámetro del halo de inhibición en la combinación cefalosporina/clavulánico con respecto a los halos de cefalosporina sin clavulánico. Las variables de este estudio fueron: Crecimiento de microorganismos (frecuencia). Resistencia a los diferentes antibióticos (sensible, resistente). Procedencia (hospitalaria y extrahospitalaria).

Las variables se expresan mediante sus frecuencias relativas en tantos por cientos (\%). Para valorar las posibles asociaciones estadísticas se utilizó la prueba exacta de Fisher, y la significación se estableció en $\mathrm{p}<0,05$.

\section{RESULTADOS}

De las 10.330 muestras procesadas durante los años 2009, 2010 y 2011 se identificaron 667 cepas de E. coli (6,46\%), 224 cepas en el 2009, 189 en 2010, y 254 en 2011. La mayoría de los aislamientos de $E$. coli estudiados provenían de infecciones urinarias 563 cepas: $(84,41 \%)$; de otras procedencias como heridas quirúrgicas 40 cepas $(6 \%)$, exudados vaginales $32(4,8 \%)$, de exudados faríngeos, muestras de contenido biliar y exudados óticos 5 cepas $(0,75 \%)$ respectivamente, de hemocultivos $7(1,05 \%)$, muestras de catéteres y otros $9(1,35 \%)$ y una de esputo $(0,15 \%)$; 104 cepas en total $(15,59 \%)$ Las cepas de E. coli detectadas y confirmadas productoras de BLEE fueron 34 lo que representa una tasa del $5,10 \%$. Se encontró una prevalencia de $2,61 \%$ en el año 2009, 6,52\% en el año 2010 y 5,98\% en el 2011 (6 cepas con BLEE en 2009, 14 en 2010 y 14 en 2011, Fig.1). 


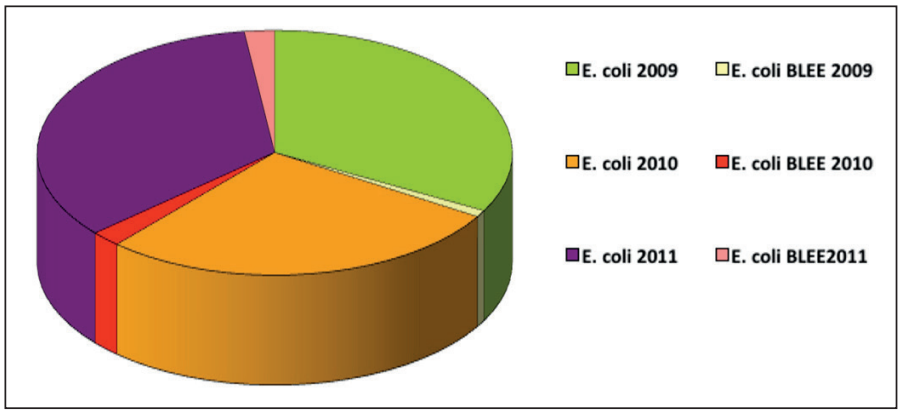

Figura 1. Número de cepas aisladas de E. coli y E. coli con BLEE en los años 2009, 2010 y 2011.

Aunque en las muestras procesadas se encontró crecimiento de cepas de Klebsiella pneumoniae ninguna de ellas fueron productoras de BLEE.

Según el origen de la infección se obtuvieron: 23 cepas en urocultivos $(67,65 \%), 8$ en heridas quirúrgicas $(23,53 \%), 2$ en exudados vaginales $(5,9 \%)$ y en esputo una cepa $(2,94 \%)$. En total, 12 cepas de origen hospitalario $(35,30 \%)$ y 22 cepas de origen extrahospitalario $(64,70 \%)$, con una mayor frecuencia procedentes de urocultivos y heridas.

En cuanto a la sensibilidad de las cepas estudiadas: todas fueron sensibles a Imipenem-Meropenem (100\%), Amoxicilina/ clavulánico el 61,75\%, Piperacilina/tazobactám el 79,40\%, Cefoxitina $94,10 \%$, Ciprofloxacino y Levofloxacino $35 \%$ ambos, Amikacina 85,3\%, Gentamicina 76,5\%, Tobramicina 61,8\%, Cotrimoxazol (SxT) 50\%, Nitrofurantoina 100\% y Fosfomicina $87 \%$ sensibles. La resistencia a las Cefalosporinas de $2^{\mathrm{a}}$ y $3^{\mathrm{a}}$ generación junto con el Aztreonam y Ampicilina fue del 100\%; Piperacilina/tazobactán el 20,6\% fue resistente, Amoxicilina/clavulánico el 38,25\%; Ciprofloxacino y Levofloxacino el 65\% en ambas, Amikacina, Gentamicina y Tobramicina 14,7\%, 23,5\%, $38,2 \%$ respectivamente, SxT 50\%, Fosfomicina 13\%, y Cefoxitina el $5,9 \%$. En la tabla 1 podemos apreciar las sensibilidades y resistencias de las cepas estudiadas a los diferentes antibióticos.

Con respecto al origen de las cepas entre hospitalarias y extrahospitalarias: Amoxicilina/clavulánico, Piperacilina/tazobactán, y Cefoxitina obtuvieron $66,67 \%, 91,67 \%$ y $100 \%$ de sensibilidad en las cepas hospitalarias y un $59,10 \%, 72,72 \%$ y $90,90 \%$ en las extrahospitalarias; Ciprofloxacino y Levofloxacino un 8,33\% en las hospitalarias y un $36,37 \%$ en las extrahospitalarias respectivamente; Amikacina, Gentamicina y Tobramicina un 83,33\%, $58,33 \%$ y $41,67 \%$ en hospitalarias y $86,36 \%, 81,82 \%$ y $72,73 \%$ en la extrahospitalarias; Fosfomicina y SxT $80 \%$ y $41,67 \%$ en las hospitalarias y un $88,90 \%$ y $54,54 \%$ en extrahospitalarias. La tabla 2 nos muestra las diferentes sensibilidades y resistencias de las cepas hospitalarias y extrahospitalarias y su porcentaje. No se ha podido establecer diferencias significativas entre las cepas hospitalarias y extrahospitalarias y la sensibilidad-resistencia a los diferentes antibióticos estudiados. Se encontró cepas con co-
Tabla 2. Cepas hospitalarias y extrahospitalarias: sensibilidades-resistencias a los distintos antibióticos y su porcentaje.

\begin{tabular}{lcccc}
\hline & \multicolumn{2}{c}{ Hospitalarias } & \multicolumn{2}{c}{ Extrahospitalarias } \\
\cline { 2 - 5 } Imipenem & $\mathbf{S}(\%)$ & $\mathbf{R}(\%)$ & $\mathbf{S}(\%)$ & $\mathbf{R}(\%)$ \\
\cline { 2 - 5 } Amox/clav. & 100 & 0 & 100 & 0 \\
Pip/tazob. & 66,67 & 33,33 & 59,10 & 40,90 \\
Cefoxitina & 91,67 & 8,33 & 72,72 & 27,28 \\
Cipro/Levo. & 100 & 0 & 90,90 & 9,10 \\
Amikacina & 8,33 & 91,67 & 36,37 & 63,63 \\
Gentamicina & 83,33 & 16,67 & 86,36 & 13,64 \\
Tobramicina & 58,33 & 41,67 & 81,82 & 18,18 \\
Fosfomicina & 41,67 & 58,33 & 72,73 & 27,27 \\
Cotrimoxazol & 80 & 20 & 88,90 & 11,10 \\
\hline
\end{tabular}

S: Sensibilidad; R: Resistencia

rresistencia a los antibióticos: Ciprofloxacino, SxT y Gentamicina; siendo la combinación de resistencia más frecuente Ciprofloxacino - SxT (32,35\%), Ciprofloxacino - SxT - Gentamicina $(14,7 \%)$ y Ciprofloxacino - Gentamicina $(8,82 \%)$.

\section{DISCUSIÓN}

La aparición de enterobacterias resistentes a cefalosporinas de amplio espectro y Aztreonam se ha convertido en un gran problema de salud pública mundial. En los últimos años se ha producido un aumento de la incidencia de enterobacterias productoras de BLEE principalmente $E$. coli.

En determinadas áreas de España y Europa se han publicado datos sobre la prevalencia de cepas productoras de BLEE, convirtiéndose en un problema creciente el aislamiento de estas cepas tanto en la comunidad como en el hospital. La frecuencia encontrada en este estudio de cepas $E$. coli con BLEE es del $5,10 \%$, un porcentaje mayor que el presentado en el proyecto GEIH-BLEE 2006 (Grupo de Estudio de la Infección Hospitalaria) donde obtuvieron el 4,4\%. En este estudio multicéntrico en hospitales españoles encontraron que el porcentaje mayor se encuentra en Andalucía, siendo Cádiz con el 20\% la que tenía la frecuencia más alta, León y Navarra las que menos 0,55\% y $0,49 \%$ respectivamente) ${ }^{6}$. En Alicante las frecuencias encontradas en dos hospitales estudiados entre los años 1999-2003 (Orihuela y Elche), fueron de 3\% y 2,25\% respectivamente ${ }^{7}$. En Málaga un estudio realizado en el año 2005 tuvo una frecuencia de $8,2 \%{ }^{2}$ y en el estudio Ena et al obtuvieron una frecuencia del 5,52\% entre los años 1999-2004. ${ }^{8}$ En otro estudio llevado a cabo en Madrid desde Enero a Diciembre de 2008, obtuvieron un $7,8 \%$ de prevalencia ${ }^{9}$. En América latina encontramos una frecuencia del 7,6 \% en un estudio realizado en Colombia entre los

Tabla 1. Sensibilidad y resistencia de las cepas E. coli con BLEE a los distintos antibióticos.

\begin{tabular}{lccccccccccc}
\hline Antibióticos & Imp & Am/cl & P/T & Cfx & Cp-Lv & Ak & Gm & Tm & Fos & SxT & Cfl/A \\
\hline Sensible (\%) & 100 & 61,76 & 79,4 & 94,1 & 35 & 85,3 & 76,5 & 61,8 & 87 & 50 & 0 \\
Resistente (\%) & 0 & 38,24 & 20,6 & 5,9 & 65 & 14,7 & 23,5 & 38,2 & 13 & 50 & 100 \\
\hline
\end{tabular}

Imp: Imipenem, Am/cl: Amoxicilina/clavulánico, P/T: Piperacilina/tazobactam, Cfx: Cefoxitina, Cp-Lv: Ciprofloxacina y Levofloxacina, Ak: Amikacina, Gm: Gentamicina, Tm: Tobramicina, Fos: Fosfomicina, SxT: Cotrimoxazol, Cfl/A: Cefalosporinas $2^{\mathrm{a}}$ y $3^{\mathrm{a}}$ generación y Aztreonam. 


\section{Escherichia coli portador de betalactamasas de espectro extendido. Resistencia}

años 2005-200910. Además, las ciudades anteriormente citadas confirman el incremento en mayor o menor grado de la prevalencia de cepas E. coli con BLEE respecto a los datos de estos estudios y otros realizados anteriormente; en este estudio también se notó un aumento con respecto a las frecuencias encontradas entre el año 2009 y el $2010(2,6 \%)$ y $(6,9 \%)$ aunque en el 2011 fue del 5,98\%; e igualmente concuerdan en que la mayoría de éstas cepas son de procedencia extrahospitalarias y de origen urinario. En la figura 2 apreciamos la frecuencia de crecimiento de $E$. coli con BLEE en este estudio y en los estudios de las ciudades mencionadas.

Asimismo EARSS (Europeam Antimicrobial Resistence Surveillance System) informa que existe una variabilidad geográfica y un aumento de cepas de E. coli resistente a cefalosporinas de amplio espectro. En el estudio llevado a cabo en el año 2005, Portugal, Chipre, Rumanía y Bulgaria tuvieron una frecuencia de $12 \%, 16 \%, 16 \%$ y $28 \%$ respectivamente; además de informar que la prevalencia en la mayor parte de Europa está entre el 1-5\%, que España se encuentra entre el 5-10\% y que Irlanda e Italia entre el $10-25 \%$. Confirman también que son más los informes en los que E. coli con BLEE se aíslan en infecciones comunitarias. El programa MYSTIC (Meropenem Yearly Susceptibility test Information Collection) también informó de un aumento de la prevalencia de cepas E. coli con BLEE en Europa, pasando del $2,1 \%$ al $10,8 \%$ entre los años 1997 y $2004^{1,6}$.

Según diferentes estudios, la resistencia se ha asociado al uso masivo de cefalosporinas de amplio espectro y quinolonas, constituyendo con ello la aparición microorganismos productores de BLEE. Pero no siempre el personal médico ha tenido en cuenta este hecho y el papel que tiene la aplicación de un correcto uso de antibióticos.

La sensibilidad de este estudio a Imipenem (100\%) coincide con los resultados de los estudios hasta ahora realizados en otras ciudades. El estudio GEIH-BLEE 2006 en España ${ }^{6}$, mostró una sensibilidad- resistencia para los diferentes antimicrobianos estudiados de: Imipenem 100\%, Amikacina el 93,5\% - 6,5\%, Amoxicilina/clavulánico 69\% - 31\%, Piperacilina/tazobactán $85 \%$ - 15\%, Cefoxitina 76,5\% - 23,5\%, Ciprofloxacino 37,5\% $62,5 \%$, Gentamicina $66 \%$ - 34\%, Tobramicina $65 \%$ - 35\% y SXT $25 \%-75 \%$ respectivamente. En este estudio, en la mayoría de

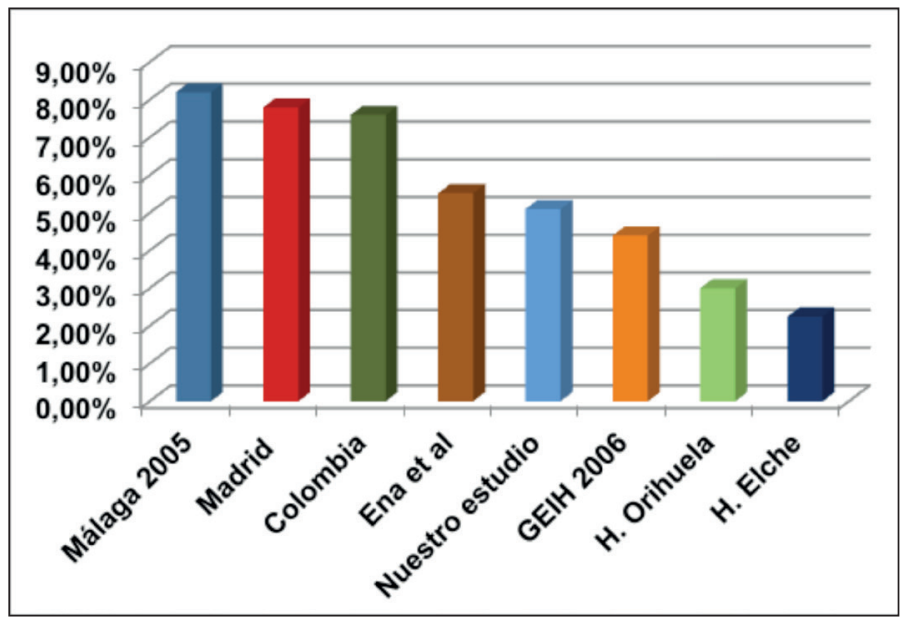

Figura 2. Frecuencia de crecimiento de E. coli con BLEE en este estudio y en las distintas ciudades. los antibióticos se obtuvo un porcentaje inferior de sensibilidad, excepto en Cefoxitina con 94,10\% - 5,90\%, Gentamicina 76,5\% - $23,5 \%$ y SXT con un $50 \%$ de sensibilidad-resistencia respectivamente. Sin embargo Colombia ${ }^{10}$ tiene un alto porcentaje de resistencia a la mayoría de antimicrobianos. En su estudio obtuvieron: Imipenem un 14,5\%, Amikacina el 19,4\%, Piperacilina/tazobactán 20,6\%, Cefoxitina 57,2\%, Ciprofloxacino 76,6\%, Gentamicina $69,3 \%$ y SXT un $72,6 \%$. En la tabla 3 podemos ver las resistencias de este estudio, de los estudios anteriormente mencionados y sus porcentajes.

Las opciones terapéuticas para las infecciones causadas por bacterias productoras de BLEE son muy limitadas. A la elevada resistencia intrínseca a betalactámicos se une la resistencia cruzada (corresistencia) a otros antibióticos no betalactámicos ${ }^{11}$; el efecto inóculo (la CMI del antibiótico aumenta 10-100 veces debido a una carga bacteriana mayor) que con frecuencia presentan, la inducción de pérdida de porinas y la sobreproducción de betalactamasas resistentes a inhibidores ${ }^{12}$.

La mayoría de autores concuerdan que Imipenem es el tratamiento de elección para las infecciones graves y pacientes de riesgo frente a cepas E. coli con BLEE. Pero aunque la resistencia es rara, se debe ser cauteloso con el empleo del mismo, ya que con su uso indiscriminado pueden aparecer cepas de bacilos gramnegativos no fermentadores multirresistentes (Acinetobacter spp, Stenotrophomonas maltophilia o Pseudomonas spp. $)^{3}$. Además ya se han descrito fenómenos de resistencia por carbapenemasas, (se ha encontrado en Barcelona dos cepas de $E$. coli y Klebsiella penumoniae $)^{14}$. Hay autores que no aconsejan Piperacilina/tazobactán como tratamiento empírico en las infecciones graves ya que puede ser menos eficaz debido al efecto inóculo, variable en infecciones sistémicas, y es necesario el estudio de sensibilidad. Cefoxitina presenta disponibilidad para su utilización pero su uso es limitado debido a la pérdida de porinas por las cuales entra el antibiótico. Amoxicilina/clavulánico no se ve afectada por el efecto inóculo; algunos autores opinan que puede ser una opción para el tratamiento de infecciones urinarias siempre que sean sensibles, pero hay escasa experiencia en infecciones sistémicas ${ }^{12}$. Otros autores no lo recomiendan de forma empírica en infecciones urinarias ya que se asocia a mayor tasa de recurrencia y se alcanzan menores concentraciones en esta localización ${ }^{11}$. Amikacina tiene menos tasa de resistencia pero tiene como inconveniente la toxicidad renal. Gentamicina y Tobramicina pueden formar parte del fenómeno de resistencia cruzada, por lo que no son recomendables en monoterapia. Ci-

Tabla 3. Resistencias (\%) de E. coli BLEE en los distintos estudios.

\begin{tabular}{lccc}
\hline & Este estudio & GEIH-BLEE & Colombia \\
\hline Imipenem & 0 & 0 & 14,5 \\
Amikacina & 14,7 & 6,5 & 19,4 \\
Amox/clav. & 38,24 & 31 & - \\
Pip/tazobac. & 20,6 & 15 & 20,6 \\
Cefoxitina & 5,88 & 23,5 & 57,2 \\
Ciprofloxacina & 65 & 62,5 & 76,6 \\
Gentamicina & 23,5 & 34 & 69,3 \\
Tobramicina & 38,2 & 35 & - \\
Cotrimoxazol & 50 & 75 & 72,6 \\
\hline
\end{tabular}


profloxacino y Levofloxacino aunque alcanzan una mayor concentración urinaria y se han utilizado con mucha frecuencia en tratamientos empíricos, presentan últimamente un porcentaje alto de resistencia por lo que es necesario el estudio de sensibilidad. Fosfomicina es el antibiótico más recomendable en infecciones urinarias, ya que presenta una tasa baja de resistencia y a su elevada concentración en dicha localización se une su fácil administración ${ }^{11}$. SxT según algunos autores es de gran eficacia pero se utiliza poco debido a su elevada resistencia.

Los resultados de las resistencias encontradas confirman la necesidad de un control en la política de uso de antibióticos. El tratamiento empírico es práctica habitual, pero para instaurarlo es importante conocer los patrones de sensibilidad antimicrobiana, especialmente de E. coli. Para ello a la hora de instaurar un tratamiento en las infecciones por $E$. coli con BLEE, se ha de tener en cuenta el tipo de paciente, el origen de la infección, la gravedad y el uso previo de antibióticos ${ }^{13}$. Asimismo entre los criterios más importantes para iniciar un tratamiento empírico también ha de tenerse en cuenta que el porcentaje de sensibilidad sea elevado y el cumplimiento terapéutico sencillo ${ }^{11}$. Como el aumento de cepas $E$. coli con BLEE constituye un importante problema en las infecciones causadas por este microorganismo tales como: bacteriemia, peritonitis o neumonía nosocomial, sería imprescindible realizar el cultivo a los pacientes con sospecha de infecciones para así conocer los patrones de resistencia, evitando con ello el uso empírico de antibióticos, fracasos terapéuticos y nuevas resistencias de cepas productoras de BLEE.

Este estudio destaca el hecho de que muchas cepas de $E$. coli con BLEE son de origen extrahospitalario. Estos resultados se deben en parte a que la mayoría de las muestras que se procesan en este laboratorio son de dicho origen y que la mayoría de las cepas productoras de BLEE se aislaron en orina, además aunque las características de este Hospital en estos años sea de una baja ocupación hospitalaria, el porcentaje de cepas encontradas es elevado, por lo que con estos resultados, se ha de tener en cuenta que aunque no refleja toda la población de riesgo y las limitaciones que presenta el ser un estudio retrospectivo; los resultados obtenidos si reflejan el comportamiento de los microorganismos de la localidad y su evolución, permitiendo conocer los patrones de sensibilidad y resistencia; siendo éstos una información necesaria tanto para el clínico como para el médico de atención primaria a la hora de instaurar un tratamiento con antimicrobianos, seleccionando la terapia apropiada en pacientes con sospecha de infecciones causadas por gérmenes productores de BLEE.

\section{CONCLUSIONES}

Tras los resultados obtenidos en este estudio se puede concluir que, la frecuencia encontrada de Escherichia coli portador de BLEE en las cepas aisladas presentan un porcentaje similar a la de otros estudios realizados en España.

La tasa de resistencia encontrada para algunos antimicrobianos como: Amoxicilina/clavulánico, Cotrimoxazol y Fluorquinolonas en las cepas estudiadas de nuestra población es elevada.

\section{ASPECTOS ETICOS}

Debido a que se trataba de un estudio retrospectivo sin riesgos para los pacientes ya que sólo se usaron los datos del Laboratorio de Microbiología, no se requirió el consentimiento informado y se mantuvo la confidencialidad de la identidad de los pacientes según las normas de la ley de protección de datos de carácter personal LO 15/1999.

\section{BIBLIOGRAFÍA}

1. García-Hernández A. M., García Vázquez, E., Hernández-Torres A., Ruiz J., Yagüe G., Herrero J. A. et al. Bacteriemias por Escherichia coli productor de betalactamasas de espectro extendido (BLEE) significación clínica y perspectivas actuales. Revista Española de Quimioterapia 2011; 24(2): 57-66

2. García López M.V., Gallardo García M.H., Rodríguez - Ortega R., Ropero Pinto F., Granados Martín E., Viciana Ramos M.I., et al. Distribución de los patrones de sensibilidad de Escherichia coli intrahospitalario y extrahospitalario y los fenotipos de resistencias asociados durante el año 2005. Revista Española de Quimioterapia 2008; 21(3): 157-165

3. Sanchez Artola, B. Betalactamasas de espectro extendido (BLEE). Curso sepsis grave: capítulo 6. Revista Electrónica de Medicina Intensiva, 2004; 4 (8), Artículo ${ }^{\circ} \mathrm{C} 6$

4. Oliver A., Cantón R. Enterobacterias productoras de B-Lactamasas plasmídicas de espectro extendido. www.seim.org/control/revisiones/bacteriología/Blee.pdf.

5. Martín Clavo S. Martín Cillero M.T. Liso Rubio F. J. Tratamiento de las infecciones producidas por beta-lactamasas de espectro extendido (BLEE). www.fundacionpromedia.org/estudios-universitarios/.../cap 4.pdf.

6. Ángel Díaz M., Ramón Hernández J., Martinez-Martinez L., RoriguezBaño J., Pascual A. Escherichia coli y Klebsiella pneumoniae productores de betalactamasas de espectro extendido en hospitales españoles: segundo estudio multicéntrico (Proyecto GEIH- BLEE 2006). Enfermedades Infecciosas y Microbiología Clínica.2009; 27 (9):503-510

7. Yague A., Cebrián L., Rodriguez-Díaz J. C., Gonzalo-Jimenez N., Royo G., Campillos, P. et al. Cepas de Escherichia coli productoras de betalactamasas de espectro extendido: origen, Características e incidencia en el sur de la provincia de Alicante en el período 1999-2003. Enfermedades Infecciosas y Microbiología Clínica. 2005; 23 (2) 76-79.

8. Ena J, Arjona F, Martinez-Peinado C, López-Perezagua MM, Amador C. Epidemiology of urinary tract infections caused by extended-spectrum B-lactamase-produciong Escherichia coli. Urology 2006; 68:1169-74

9. Moreno M.J., Alarcón T., Domingo D., Agudo S., Martinez M.C. y LópezBrea M. Enterobacterias portadoras de BLEE aisladas de muestras clínicas de acuerdo con el área de procedencia del paciente (Hospital o Atención primaria). XIII Reunión de la Sociedad Española de Enfermedades Infecciosas y Microbiología Clínica (SEIM) 2007.

10. Pérez N., Pavas N., Rodriguez E. I. Resistencia a los antibióticos en Escherichia coli con beta-lactamasas de espectro extendido en un hospital de la Orinoquia colombiana. Infectio. 2011; 15(3): 147-154.

11. Tena D., Glez-Praetorius A., González J. C., Heredero E., Illescas S., Sainz de Baranda C., et al. Evolución del patrón de sensibilidad de Escherichia coli en infecciones del tracto urinario diagnosticados en la comunidad durante el período 2003-2007. Estudio multicéntrico en Castilla la Mancha. Revista Española de Quimioterapia 2010, 23(1):36-42.

12. Blanco C. Tratamiento de infecciones graves por gérmenes productores de BLEE. UCI del Hospital Son Dureta. wwwelcomprimido.com/Blanco_tratamientoBLEE_INfecgrave

13. Gómez Gómez, J. Infección urinaria por Escherichia coli multirresistente: impacto clínico y nuevas perspectivas. Medicina Clínica . 2007; 129(11):412-3

14. Baquero F., Cercenado E., Cisterna R., de la Rosa M., García-Rodriguez J.A., Gobernado M., et al. Patrones de sensibilidad a antimicrobianos de Enterobacteriaceas causantes de infecciones intraabdominales en España: resultados del estudio SMART 2003. Rev. Esp. Quimioterap. 2006; 19 (1): 51-59 